\title{
Cognitive function predicts work disability among multiple sclerosis patients
}

\author{
Andrius Kavaliunas (D), Petter Tinghög, Emilie Friberg, Tomas Olsson, Kristina Alexanderson, \\ Jan Hillert and Virginija Danylaite Karrenbauer
}

\section{Abstract}

Background: In multiple sclerosis various aspects of cognitive function can be detrimentally affected. More than that, patients' employment and social functioning is likely to be impacted.

Objective: To determine whether work disability among multiple sclerosis patients could be predicted by the symbol digit modalities test.

Methods: A register-based cohort study was conducted. Individual data on work disability, operationalised as annual net days of sickness absence and/or disability pension were retrieved at baseline, when the symbol digit modalities test was performed, after one-year and 3-year follow-up for 903 multiple sclerosis patients. The incidence rate ratios for work disability were calculated with general estimating equations using a negative binomial distribution and were adjusted for gender, age, educational level, family composition, type of living area and physical disability.

Results: After one year of follow-up, the patients in the lowest symbol digit modalities test quartile were estimated to have a $73 \%$ higher rate of work disability when compared to the patients in the highest symbol digit modalities test quartile (incidence rate ratio $1.73,95 \%$ confidence interval 1.42-2.10). This estimate after 3-year follow-up was similar (incidence rate ratio 1.68, 95\% confidence interval 1.40-2.02).

Conclusion: Cognitive function is to a high extent associated with multiple sclerosis patients' future work disability, even after adjusting for other factors.

Keywords: Multiple sclerosis, cognition, work, employment, prognosis, socioeconomic factors

Date received: 20 April 2018; Revised received 8 November 2018; accepted: 22 November 2018

\section{Introduction}

Approximately 2.5 million people worldwide are affected with multiple sclerosis (MS), a chronic neuroinflammatory disease of the brain and spinal cord that is a common cause of serious physical disability in young adults. ${ }^{1}$ MS poses a major personal and socioeconomic burden: the average age of disease onset is 30 years - a time that is decisive for work and family planning - and 25 years after diagnosis approximately $50 \%$ of patients require permanent use of a wheelchair. The condition has a heterogeneous presentation that can include sensory and visual disturbances, motor function impairments, fatigue, pain and cognitive deficits. ${ }^{1,2} \mathrm{MS}$ is associated with reduction in work capacity and lower earnings. ${ }^{3}$ It causes work disability and healthcare resource use - the estimated cost of illness of all the MS patients in Sweden in 2010 was SEK3950 million, of which $75 \%$ was indirect costs (the productivity losses, identified from sick-leave benefits and disability pension (DP) benefits). ${ }^{4}$ In a recent study, the rate of MS patients of working age who were on DP was more than $60 \%,{ }^{5}$ highly elevated compared to the equivalent general population.

Cognitive dysfunction is present in up to $70 \%$ of patients with MS. $^{6}$ Various aspects of cognitive function can be detrimentally affected: difficulties with long-term and verbal memory as well as with
Multiple Sclerosis JournalExperimental, Translational and Clinical

January-March 2019, 1-8

DOI: $10.1177 /$ 2055217318822134

(C) The Author(s), 2019 Article reuse guidelines: sagepub.com/journalspermissions
Correspondence to: Andrius Kavaliunas, Department of Clinical Neuroscience, Karolinska Institutet, Tomtebodavägen 18A:05, Stockholm, 17177 Sweden

andrius.kavaliunas@ki.se, Twitter:@andriuskava

Andrius Kavaliunas, Department of Clinical Neuroscience, Karolinska Institutet, Sweden

Petter Tinghög, Department of Clinical

Neuroscience, Karolinska Institutet, Sweden Red Cross University College, Sweden 
Emilie Friberg,

Department of Clinical

Neuroscience, Karolinska

Institutet, Sweden

Tomas Olsson,

Department of Clinical

Neuroscience, Karolinska

Institutet, Sweden

Center for Molecular

Medicine, Karolinska

University Hospital, Sweden

Kristina Alexanderson,

Department of Clinical

Neuroscience, Karolinska

Institutet, Sweden

Jan Hillert,

Department of Clinical

Neuroscience, Karolinska

Institutet, Sweden

Center for Molecular

Medicine, Karolinska

University Hospital, Sweden

Department of Neurology,

Karolinska University

Hospital, Sweden

Virginija Danylaite

Karrenbauer,

Department of Clinical

Neuroscience, Karolinska

Institutet, Sweden

Department of Neurology,

Karolinska University

Hospital, Sweden abstract and conceptual reasoning, fluency, planning, visuospatial perception and reduced speed of information processing. ${ }^{6}$ Information processing speed is the very first cognitive deficit that emerges and one of the domains in which cognitive impairment is most marked in MS. ${ }^{7,8}$ It is also considered as a primer for and predictor of the future impairment of other cognitive domains such as memory. One of the widely used tests of processing efficiency and speed in MS is the symbol digit modalities test (SDMT). ${ }^{9}$ It is more congenial for both patient and assessor, takes less time to complete and has equal psychometric validity compared to other tests of attention and processing speed, for example, the paced auditory serial addition task, and is recommended as a clinical tool for neurologists and healthcare professionals working with MS patients. ${ }^{10}$ The research in MS clearly supports the reliability and validity of the test, ${ }^{11}$ which is sensitive, specific and an accurate tool to classify cognitive impairment, ${ }^{12,13}$ and has been shown to be the best predictor of MS cognitive impairment in both the brief repeatable battery of neuropsychological tests and the minimal assessment of cognitive function in MS. ${ }^{14}$ The test has been used in a Swedish nationwide post-marketing surveillance study of new MS treatments. $^{15}$

Some studies have reported that cognitive difficulties involve problems with paid work, and the impact of MS on work productivity and its possible associations with not being employed have recently attracted great interest. ${ }^{16,17}$ For example, Kobelt et al. ${ }^{18}$ reported that regular work hours decreased linearly with increasing severity of fatigue and cognitive problems. Also, a recent study by Björkenstam et al. showed that there is a considerable heterogeneity of MS progression in terms of sickness absence (SA) and DP. ${ }^{19}$

While many clinical and demographic factors have frequently been associated with work disability, few studies have examined whether there are predictors of future disability, even over the short term. ${ }^{20}$ Thus, in this study we aimed to determine whether the SDMT can be used to predict work disability among MS patients.

\section{Materials and methods}

A longitudinal, register-based cohort study was conducted, using data from the following three nationwide sources:
1. The clinically generated Swedish Multiple Sclerosis Register (SMSreg) was used to obtain information about individuals diagnosed with MS, including the scores of the Expanded Disability Status Scale (EDSS) and SDMT, which has been used in a Swedish nation-wide post-marketing surveillance study of new MS treatments. $^{15,21}$ SMSreg runs on government funding and is used in all Swedish neurology departments. Currently the SMSreg includes data on 16,600 of Sweden's estimated 20,700 patients with $\mathrm{MS}^{22}$

2. The Micro Data for Analysis of the Social Insurance (MiDAS) database held by the National Social Insurance Agency regarding data on SA and DP.

3. The Longitudinal Integration Database for Health Insurance and Labour Market Studies (LISA) held by Statistics Sweden regarding information on sociodemographic variables (gender, age, family composition, type of living area and education). ${ }^{23}$

The unique personal identification number assigned to all residents in Sweden was used to conduct the linkage of data at an individual level.

MS patients aged 20-62 years who lived in Sweden and had a clinical visit with SDMT recorded throughout 2006-2009 were identified from the SMSreg. In the SDMT, the patient is presented with nine graphical symbols, each paired with a single digit, serving as a key. Below are rows of the symbols, randomly ordered, and the patient must say the numbers that go with each digit. Besides the SDMT, we were able to track the grade of disability, which is routinely quantified by neurologists according to the EDSS. ${ }^{24}$ The EDSS spans between 0 and 10 with increments of 0.5 in which 0 is a neurologically unaffected patient and 10 is death as a result of MS.

The sociodemographic information at the year of the first SDMT recorded (T0) was added from LISA. In addition, the patients had to be of working age, that is older than 19 years at baseline (T0) and under 65 years at T3. Individual data on work disability, operationalised as annual days of SA and/or DP was retrieved from MiDAS at baseline (T0), after oneyear (T1) and 3-year (T3) follow-up. Net days were calculated combining part-time absence days to full days, for example, two gross days with $50 \%$ absence were calculated as one net day. In the analysis, type of living area was categorised into: (a) larger cities 
(Stockholm, Gothenburg, and Malmö); (b) mediumsized municipalities (with more than 90,000 inhabitants within $30 \mathrm{~km}$ distance from the centre of the city); (c) smaller municipalities. The family composition was categorised into two types: married/ cohabiting (living with a partner) and single.

In total, $903 \mathrm{MS}$ patients were included in the study.

\section{$S A$ and DP in Sweden}

In Sweden, people with an income from work or unemployment benefits who have a reduced work capacity due to disease or injury can be granted SA benefits. For most employees the first 14 days of a SA spell is paid by the employer, after that by the Social Insurance Agency. All people aged 19-64 years can be granted DP if their work incapacity, caused by disease or injury, is long term or permanent. Both SA and DP can be granted for full time or part time $(25 \%, 50 \%$ or $75 \%)$ of ordinary work hours.

\section{Statistical analyses}

Descriptive statistics with means, medians and proportions were used to describe the cohort at baseline. One-way analysis of variance (ANOVA) was used to compare continuous variables across SDMT quartiles. For the categorical variables a chi-square test was used, for medians a Kruskal-Wallis test was used.

Incidence rate ratios (IRRs) for work disability, crude and adjusted for gender, age, education, family composition, type of living area and physical disability, were calculated with general estimating equations (GEEs) using a negative binomial distribution and autoregressive covariance matrix. Marginal means (the mean response for each factor, adjusted for all covariates in the model) of work disability at T1 and T3 for MS patient groups were estimated.

For analysis purposes, MS patients were categorised into quartiles (QI-QIV) according to their raw SDMT score (the patients with the lowest cognitive function in the first SDMT quartile (QI) and the patients with the best cognitive function in the fourth quartile (QIV)). The SDMT score was also studied as a continuous variable in a complementary analysis.

\section{Ethics}

The project was approved by the regional ethical review board of Stockholm. All Swedish residents are automatically included in the MiDAS and LISA.
Data collection into SMSreg is based on informed consent from the individual patients.

\section{Results}

Descriptive data of the study population categorised by SDMT quartiles are presented in Table 1 . Of the 903 MS patients, $71.5 \%$ were women, $43.7 \%$ had higher education (university or university college studies) and the mean age of the patients was 37.4 \pm 9.3 years. The majority of the patients were married/cohabiting $(51.2 \%)$ and living in larger cities $(50.7 \%)$. The median EDSS was 3.0 (the interquartile range 2.5) and work disability, operationalised as annual days of SA and/or DP, at baseline (T0) was on average 164 days.

MS patients were rather different when looking across SDMT quartiles (Table 1); for example, MS patients with the best cognitive function in the fourth quartile (QIV) when compared to the patients with the lowest cognitive function in the first quartile (QI) were on average younger (34.3 and 40.1 years, respectively) and displayed the lowest proportion of patients with lower education $(42.4 \%$ and $65.2 \%$, respectively). Furthermore, they were less disabled (median EDSS 2.0 and 4.0, respectively) and had lower levels of work disability (98.5 and 229.9 days per annum, respectively). A noticeable gradual change through quartiles was also apparent in many of the above-mentioned patient characteristics, e.g. decrease of mean age (40.1 years in QI, 36.4 in QIII, 38.5 in QII and 34.3 in QIV), or decrease of median EDSS (4.0 in QI, 3.5 in QII, 3.0 in QIII and 2.0 in QIV).

There were also some similarities, in which MS patients did not differ significantly across SDMT quartiles, for example, by gender proportions and family composition (Table 1).

Crude IRRs for work disability after one-year follow-up were 2.44 (95\% confidence interval (CI) 2.04-2.92) for the QI patients, 1.91 (95\% CI 1.592.28) for the QII patients and 1.52 (95\% CI $1.26-$ 1.82) for the QIII patients when compared to the QIV patients. The crude IRRs after 3 years of follow-up were $2.42(95 \%$ CI 2.04-2.89), 1.89 (95\% CI 1.59-2.24) and 1.41 (95\% CI 1.18-1.69), respectively. Adjusted IRRs for work disability among MS patients are shown in Table 2. Evident from the table, the adjusted IRR increased with worse cognitive function (lower SDMT quartile). After one year of follow-up, the QI patients were estimated to have an increased risk of work 
Table 1. Descriptive data of the study population, by SDMT quartiles.

\begin{tabular}{|c|c|c|c|c|c|}
\hline \multirow[b]{2}{*}{ Patient characteristics } & \multirow[b]{2}{*}{ All $(N=903)$} & \multicolumn{4}{|c|}{ SDMT quartiles } \\
\hline & & $\begin{array}{l}\text { QI }(0-39) \\
(n=233)\end{array}$ & $\begin{array}{l}\text { QII }(40-48) \\
(n=232)\end{array}$ & $\begin{array}{l}\text { QIII (49-56) } \\
(n=214)\end{array}$ & $\begin{array}{l}\text { QIV (57-86) } \\
(n=224)\end{array}$ \\
\hline \multicolumn{6}{|l|}{ Gender } \\
\hline Men & $257(28.5 \%)$ & $76(32.6 \%)$ & $66(28.5 \%)$ & $62(29.0 \%)$ & $53(23.7 \%)$ \\
\hline Women & $646(71.5 \%)$ & $157(67.4 \%)$ & $166(71.5 \%$ & $152(71.0 \%)$ & $171(76.3 \%)$ \\
\hline Age $(\text { mean } \pm \mathrm{SD})^{*}$ & $37.4 \pm 9.3$ & $40.1 \pm 9.7$ & $38.5 \pm 8.8$ & $36.4 \pm 9.1$ & $34.3 \pm 8.6$ \\
\hline \multicolumn{6}{|l|}{ Education? } \\
\hline Lower and secondary & $508(56.3 \%)$ & $152(65.2 \%)$ & $148(63.8 \%)$ & $113(52.8 \%)$ & 95 (42.4\%) \\
\hline Higher & $395(43.7 \%)$ & $81(34.8 \%)$ & $84(36.2 \%)$ & $101(47.2 \%)$ & $129(57.6 \%)$ \\
\hline \multicolumn{6}{|l|}{ Family composition } \\
\hline Married/cohabiting & $462(51.2 \%)$ & $110(47.2 \%)$ & $128(55.2 \%)$ & $106(49.5 \%)$ & $118(52.7 \%)$ \\
\hline Single & $441(48.8 \%)$ & $123(52.8 \%)$ & $104(44.8 \%)$ & $108(50.5 \%)$ & $106(47.3 \%)$ \\
\hline \multicolumn{6}{|l|}{ Type of living area } \\
\hline Larger cities & $458(50.7 \%)$ & $141(60.5 \%)$ & 109 (47.0\%) & $98(45.8 \%)$ & $110(49.1 \%)$ \\
\hline $\begin{array}{l}\text { Medium-sized } \\
\text { municipalities }\end{array}$ & $256(28.4 \%)$ & 47 (20.2\%) & $66(28.5 \%)$ & $74(34.6 \%)$ & $69(30.8 \%)$ \\
\hline Smaller municipalities & $189(20.9 \%)$ & 45 (19.3\%) & $57(24.6 \%)$ & $42(19.6 \%)$ & $45(20.1 \%)$ \\
\hline EDSS (median (IQR))** & $3.0(2.5)$ & $4.0(3.0)$ & $3.5(2.75)$ & $3.0(1.5)$ & $2.0(2.0)$ \\
\hline Work disability at $\mathrm{T} 0^{*}$ & 164.0 & 229.9 & 182.2 & 141.2 & 98.5 \\
\hline $\begin{array}{l}\text { SDMT: symbol digit modalit } \\
\text { quartile range. } \\
{ }^{*} P<0.001 \text {, one-way analysis } \\
{ }^{* *} P<0.001 \text {, Kruskal-Wallis } \\
{ }^{\mathbb{P}} P<0.05 \text {, chi-square test. }\end{array}$ & $\begin{array}{l}\text { es test; EDSS: E } \\
\text { of variance. }\end{array}$ & anded Disabilit & Status Scale; S & : standard deviat & n; IQR: inter- \\
\hline
\end{tabular}

disability days by $73 \%$ when compared to the QIV patients (IRR 1.73, 95\% CI 1.42-2.10). This estimate after 3 years follow-up was similar (IRR 1.68, 95\% CI 1.40-2.02).

Physical disability, assessed with the EDSS, turned out to be one of the most significant factors in our analysis, with the highest IRR of work disability for the most disabled patient group. Their IRR was more than doubled when compared to MS patients with mild physical disability (IRR 2.42 at T1 and 2.61 at T3). Other significant factors were lower and secondary education (IRR 1.78 at $\mathrm{T} 1$ and 1.77 at T3 when compared to those with higher education), female gender (IRR 1.62 at T1 and 1.55 at T3) and older age (1.53 at $\mathrm{T} 1$ and 1.70 at $\mathrm{T} 3$ for the oldest patient group).

In the estimated marginal means analysis, SDMT performance at T0 predicted 247 mean annual days of work disability one year later (T1) and 259 days 3 years later (T3) for the QI patients. A total of 143 annual days of work disability at T1 and 154 days at T3 were predicted for the QIV patients (Figure 1).
The gradual change of the predicted work disability through quartiles was also apparent.

\section{Discussion}

In this cohort study, based on three nation-wide registries, we investigated how cognitive function, assessed with the SDMT, predicts works disability, operationalised as future annual net days of SA and/ or DP among MS patients. We found substantial differences in short and long-term work disability across different MS patient groups when categorised by SDMT quartiles. At baseline, MS patients in the lowest quartile had twice as much work disability as the patients in the highest quartile (229.9 and 98.5 days per annum, respectively). After one year of follow-up, the QI patients were estimated to have $73 \%$ more annual days of SA/DP when compared to the QIV patients (IRR 1.73, 95\% CI 1.42-2.10); similarly, after 3 years of follow-up (IRR 1.68, 95\% CI 1.40-2.02). This might have great implications on household income and quality of life. For most people, work is salient to life, is central to wellbeing, and is a means by which individuals define 
Table 2. Adjusted incidence rate ratios for work disability among MS patients.

\begin{tabular}{|c|c|c|c|c|}
\hline \multirow[b]{2}{*}{ Factors } & \multicolumn{2}{|l|}{$\mathrm{T} 1$} & \multicolumn{2}{|l|}{$\mathrm{T} 3$} \\
\hline & IRR & $95 \% \mathrm{CI}$ & IRR & $95 \% \mathrm{CI}$ \\
\hline \multicolumn{5}{|l|}{ SDMT quartiles } \\
\hline I & 1.73 & $1.42-2.10$ & 1.68 & $1.40-2.02$ \\
\hline II & 1.41 & $1.18-1.70$ & 1.33 & $1.12-1.58$ \\
\hline III & 1.33 & $1.11-1.60$ & 1.22 & $1.03-1.45$ \\
\hline IV & Reference & Reference & Reference & Reference \\
\hline \multicolumn{5}{|l|}{ Gender } \\
\hline Men & Reference & Reference & Reference & Reference \\
\hline Women & 1.62 & $1.40-1.86$ & 1.55 & $1.36-1.77$ \\
\hline \multicolumn{5}{|l|}{ Age groups (years) } \\
\hline $20-34$ & Reference & Reference & Reference & Reference \\
\hline $35-44$ & 1.34 & $1.15-1.56$ & 1.37 & $1.19-1.58$ \\
\hline $45-54$ & 1.48 & $1.23-1.78$ & 1.50 & $1.27-1.79$ \\
\hline $55-62$ & 1.56 & $1.08-2.24$ & 1.70 & $1.21-2.40$ \\
\hline \multicolumn{5}{|l|}{ Education } \\
\hline Lower and secondary & 1.78 & $1.56-2.04$ & 1.77 & $1.57-2.01$ \\
\hline Higher & Reference & Reference & Reference & Reference \\
\hline \multicolumn{5}{|l|}{ Family composition } \\
\hline Married/cohabiting & Reference & Reference & Reference & Reference \\
\hline Single & 0.96 & $0.84-1.09$ & 0.93 & $0.82-1.05$ \\
\hline \multicolumn{5}{|l|}{ Type of living area } \\
\hline Larger cities & Reference & Reference & Reference & Reference \\
\hline Medium-sized municipalities & 1.15 & $0.99-1.34$ & 1.21 & $1.05-1.39$ \\
\hline Smaller municipalities & 1.30 & $1.09-1.54$ & 1.38 & $1.17-1.62$ \\
\hline \multicolumn{5}{|l|}{ EDSS } \\
\hline Mild $(0-3.5)$ & Reference & Reference & Reference & Reference \\
\hline Moderate mild (4-5.5) & 1.78 & $1.49-2.12$ & 1.88 & $1.59-2.22$ \\
\hline Moderate severe (6-6.5) & 2.08 & $1.69-2.55$ & 2.23 & $1.84-2.70$ \\
\hline Severe $(7-9.5)$ & 2.42 & $1.72-3.39$ & 2.61 & $1.90-3.60$ \\
\hline \multicolumn{5}{|c|}{$\begin{array}{l}\text { MS: multiple sclerosis; CI: confidence interval; IRR: incidence rate ratio; SDMT: symbol digit modalities test; EDSS: } \\
\text { Expanded Disability Status Scale. } \\
\text { Estimates for the T1 and T3 models in the table are also adjusted for the calendar year when the SDMT } \\
\text { was performed. } \\
\text { In the adjusted model with SDMT as the continuous variable, IRRs were } 0.988 \text { (95\% CI } 0.984-0.993 \text { ) and } 0.988 \text { ( } 95 \% \\
\text { CI 0.984-0.992) for T1 and T3, respectively. }\end{array}$} \\
\hline
\end{tabular}

themselves, thus employment may be regarded as a marker of overall functioning of the individual patient. ${ }^{25}$ Furthermore, the effect of cognitive dysfunction on the social and working life of MS patients is still underestimated as MS is widely viewed as producing neurological defects primarily in the motor sphere. ${ }^{26}$

We have previously assessed MS patients' income in relation to physical disability, ${ }^{27}$ cognitive function ${ }^{28}$ and disease phenotype, ${ }^{29}$ and showed that cognitive function affects the financial situation negatively, independently of physical disability. MS patients in the highest SDMT score quartile earned more than twice the amount annually compared to patients in the lowest quartile, whereas patients in the lowest quartile received three times more income through social benefits. ${ }^{28}$ Most studies on cognitive impairment in MS are cross-sectional in nature, ${ }^{26}$ and compared to other similar studies ${ }^{30-32}$ this study has several important strengths: (a) longitudinal design; (b) a relatively large sample; (c) population-based register approach. It also contributes to other studies of socioeconomic factors in MS by exploring a new outcome measure - work disability, operationalised as annual net days of SA and/or DP. As both 


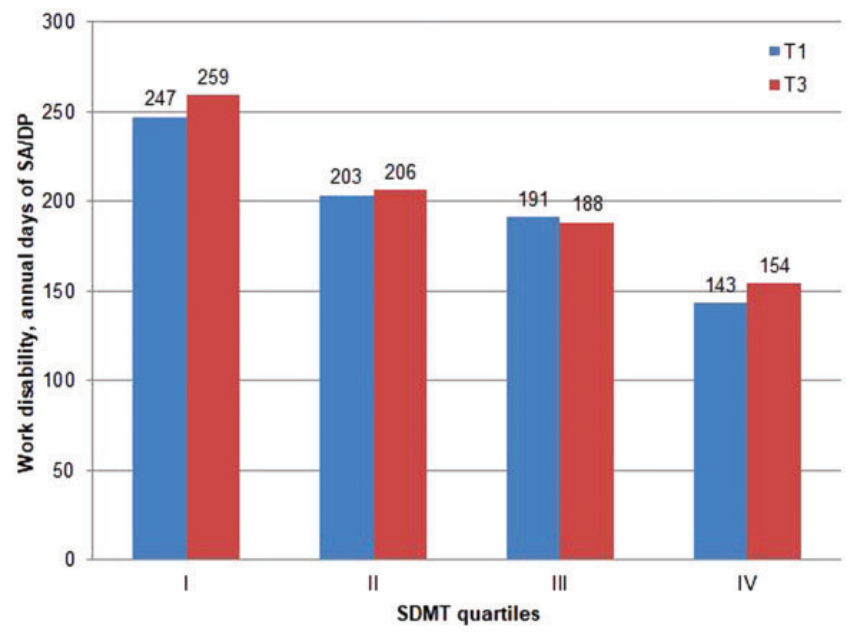

Figure 1. Predicted marginal means of work disability among multiple sclerosis patients

part-time SA and DP are possible in Sweden, it is an advantage that net days could be calculated. Moreover, compared to other measures/outcomes, SA and DP offer a continuous variable that can be assigned to every individual for each time period without missing data. ${ }^{19}$ We were also able to adjust the analyses by various important factors, in particular educational level, gender and physical disability. An interesting aspect that has also arisen from our results is the possible association of the EDSS and the SDMT - as MS patients in the highest SDMT quartile had lower EDSS scores, i.e. a median of 2.0, whereas the patients in the lowest SDMT quartile had the median EDSS score of 4.0. Whether these measures are of different construct or reflect disease progression in a similar way, as well as how they change through the clinical course in relation to each other, might be well explored in future studies.

Nevertheless, our study has to be assessed against selection bias. The SDMT, although being a widely accepted clinical tool, is not used in a daily neurology practice the same way as, for instance, the EDSS. Most of these patients underwent SDMTs because of their inclusion in the Immunomodulation and MS Epidemiology Study (IMSE) to monitor all newer MS drugs in Sweden since $2006 .{ }^{33}$ Thus, our study population represents more those who due to various reasons were treated or switched to second-line treatments or discontinued disease-modifying drugs. However, the differences of the study population in the SDMT quartiles that we observed are clear and likely to be robust. We also could not control the analysis for the form of SDMT administration, as the oral in contrast to the written form is known to give slightly higher scores. ${ }^{34}$ Another limitation is that SA days in most SA spells shorter than 15 days were not included.

MS has a detrimental impact on affected patients and a considerable economic burden of disease to society, e.g. on average during follow-up post-diagnosis MS patients had $€ 5130$ less gross salary per year compared with controls. ${ }^{35}$ A recent study showed that in spite of widespread access to modern healthcare including disease-modifying drugs, the majority of MS patients of working age were on a DP (namely, 61.7\% of the MS patients were on partial or full DP compared to $14.2 \%$ among the controls). ${ }^{5}$ Our study contributes by comprehensive analysis of various clinical and sociodemographic factors, associated with work disability, and emphasising the importance of cognitive function. Also, studying work disability may enhance the understanding of the consequences of living with chronic disease, and give new insights into the effects of sickness insurance policy in a society. ${ }^{36}$

In line with other studies, we also showed that physical disability (after adjustment), education, gender and age were significant factors to impact patients' future work disability. For example, Lunde et al. ${ }^{30}$ demonstrated that highly educated MS patients had more than a twofold chance of being employed compared to patients with less education. Findling et al. $^{31}$ reported that even with minimal disability level, a significant proportion of the studied patients had reduced work capacity. In a study by Pfleger et al., ${ }^{20}$ the hazard of being granted DP for men was $73 \%$ that of women. In addition, in our previous 
study $^{28}$ we showed gender to be such a significant factor as to impact individuals' annual earnings by SEK100,000 ( $€ € 10,500$ more for men than women; adjusted for a number of various clinical and sociodemographic variables, including age, education and SDMT). Campbell et al. ${ }^{32}$ concluded that cognitively impaired MS patients exhibited significantly lower rates of employment, and the SDMT was the most significant predictor of not being in paid work.

Finaly, by showing how cognitive function is associated with work disability of MS patients, we emphasise the necessity of testing cognition in healthcare services for MS patients. The SDMT is a simple and time-effective screening instrument for cognitive impairment and could be used as a potential tool to identify MS patients who are at high risk of short and long-term work disability in terms of SA and/or DP.

\section{Conflict of interest}

The author(s) declared the following potential conflicts of interest with respect to the research, authorship, and/or publication of this article: AK declares that there is no conflict of interest. PT and EM were funded from an unrestricted research grant from Biogen. TO has received honoraria for advisory boards and/or lecture fees, as well as unrestricted MS research grants from Biogen, Novartis and Genzyme. His MS research is funded by the Swedish Research Council, Knut and Alice Wallenberg foundation, the AFA foundation and AstraZeneca science for life grant. KA has received unrestricted research grants from Biogen and from the Swedish Research Council for Working Life, Health and Welfare. JH received honoraria for serving on advisory boards for Biogen and Novartis and speaker's fees from Biogen, MerckSerono, BayerSchering, Teva and SanofiGenzyme. He has served as principal investigator for projects sponsored by, or received unrestricted research support from Biogen, SanofiGenzyme, MerckSerono, TEVA, Novartis and BayerSchering. His MS research is funded by the Swedish Research Council and the Swedish Brain Foundation. VDK has received financial support from Stockholm County Council and Biogen's Multiple Sclerosis Registries Research Fellowship Program.

\section{Funding}

The author(s) disclosed receipt of the following financial support for the research, authorship, and/or publication of this article: The study was financially supported by the Swedish Research Council for Health, Working Life and
Welfare and by Biogen. Biogen courteously reviewed the manuscript and provided feedback to the authors. The authors had full editorial control and provided approval to the final content.

\section{ORCID iD}

Andrius Kavaliunas (D) http://orcid.org/0000-00033896-7332

\section{References}

1. Compston A and Coles A. Multiple sclerosis. Lancet 2008; 372: 1502-1517.

2. Dendrou CA, Fugger $\mathrm{L}$ and Friese MA. Immunopathology of multiple sclerosis. Nat Rev Immunol 2015; 15: 545-558.

3. Wiberg M, Friberg E, Stenbeck M, et al. Sources and level of income among individuals with multiple sclerosis compared to the general population: a nationwide population-based study. Mult Scler 2015; 21: $1730-1741$.

4. Gyllensten H, Wiberg M, Alexanderson K, et al. Costs of illness of multiple sclerosis in Sweden: a population-based register study of people of working age. Eur J Health Econ 2018; 19: 435-446.

5. Tinghog P, Hillert J, Kjeldgard L, et al. High prevalence of sickness absence and disability pension among multiple sclerosis patients: a nationwide population-based study. Mult Scler 2013; 19: 1923-1930.

6. Toosy A, Ciccarelli O and Thompson A. Symptomatic treatment and management of multiple sclerosis. Handbook Clin Neurol 2014; 122: 513-562.

7. Corfield F and Langdon D. A systematic review and meta-analysis of the Brief Cognitive Assessment for Multiple Sclerosis (BICAMS). Neurol Ther 2018; 7: 287-306.

8. Van Schependom J, D'Hooghe M B, Cleynhens K, et al. Reduced information processing speed as primum movens for cognitive decline in MS. Mult Scler 2015; 21: 83-91.

9. Langdon DW. Cognition in multiple sclerosis. Curr Opin Neurol 2011; 24: 244-249.

10. Langdon DW, Amato MP, Boringa $J$, et al. Recommendations for a Brief International Cognitive Assessment for Multiple Sclerosis (BICAMS). Mult Scler 2012; 18: 891-898.

11. Benedict RHB, DeLuca J, Phillips G, et al. Validity of the Symbol Digit Modalities Test as a cognition performance outcome measure for multiple sclerosis. Mult Scler J 2017; 23: 721-733.

12. Deloire MS, Bonnet MC, Salort E, et al. How to detect cognitive dysfunction at early stages of multiple sclerosis? Mult Scler 2006; 12: 445-452.

13. Amato MP, Portaccio E, Goretti B, et al. Relevance of cognitive deterioration in early relapsing-remitting MS: a 3-year follow-up study. Mult Scler 2010; 16: $1474-1482$. 
14. Strober L, Englert J, Munschauer F, et al. Sensitivity of conventional memory tests in multiple sclerosis: comparing the Rao Brief Repeatable Neuropsychological Battery and the Minimal Assessment of Cognitive Function in MS. Mult Scler 2009; 15: 1077-1084.

15. Holmen C, Piehl F, Hillert J, et al. A Swedish national post-marketing surveillance study of natalizumab treatment in multiple sclerosis. Mult Scler 2011; 17: 708-719.

16. Incerti $\mathrm{CC}$, Argento $\mathrm{O}$, Magistrale $\mathrm{G}$, et al. Adverse working events in patients with multiple sclerosis. Neurol Sci: official journal of the Italian Neurological Society and of the Italian Society of Clinical Neurophysiology 2017; 38: 349-352.

17. Schiavolin S, Leonardi M, Giovannetti AM, et al. Factors related to difficulties with employment in patients with multiple sclerosis: a review of 20022011 literature. Int $J$ Rehabil Res/Internationale Zeitschrift fur Rehabilitationsforschung/Revue internationale de recherches de readaptation 2013; 36: $105-111$.

18. Kobelt G, Langdon D and Jönsson L. The effect of self-assessed fatigue and subjective cognitive impairment on work capacity: the case of multiple sclerosis. Mult Scler 2018; Epub ahead of print 17 April 2018. DOI: $10.1177 / 1352458518769837$

19. Björkenstam C, Alexanderson K, Wiberg M, et al. Heterogeneity of sickness absence and disability pension trajectories among individuals with MS. Mult Scler J Exp Transl Clin 2015; 1: 1-11

20. Pfleger CC, Flachs EM and Koch-Henriksen N. Social consequences of multiple sclerosis: clinical and demographic predictors - a historical prospective cohort study. Eur J Neurol: the official journal of the European Federation of Neurological Societies 2010; 17: 1346-1351.

21. Matell H, Lycke J, Svenningsson A, et al. Age-dependent effects on the treatment response of natalizumab in MS patients. Mult Scler 2015; 21: 48-56.

22. Hillert J and Stawiarz L. The Swedish MS registry clinical support tool and scientific resource. Acta Neurol Scand Suppl. 2015; 132: 11-19.

23. Statistics Sweden. Integrated database for labour market research: Background facts, labour and education statistics 2011. Örebro: Statistics Sweden (SCB), 2011.

24. Kurtzke JF. Rating neurologic impairment in multiple sclerosis: an expanded disability status scale (EDSS). Neurology 1983; 33: 1444-1452.
25. Pack TG, Szirony GM, Kushner JD, et al. Quality of life and employment in persons with multiple sclerosis. Work - a Journal of Prevention Assessment \& Rehabilitation 2014; 49: 281-287.

26. Amato MP, Ponziani G, Siracusa G, et al. Cognitive dysfunction in early-onset multiple sclerosis: a reappraisal after 10 years. Arch Neurol 2001; 58: 1602-1606.

27. Kavaliunas A, Wiberg M, Tinghog P, et al. Earnings and financial compensation from social security systems correlate strongly with disability for multiple sclerosis patients. PloS One. 2015; 10: e0145435.

28. Kavaliunas A, Danylaite Karrenbauer V, Gyllensten $\mathrm{H}$, et al. Cognitive function is a major determinant of income among multiple sclerosis patients in Sweden acting independently from physical disability. Mult Scler 2017: Epub ahead of print 16 November 2017. DOI: $10.1177 / 1352458517740212$

29. Kavaliunas A, Manouchehrinia A, Danylaite Karrenbauer $\mathrm{V}$, et al. Income in multiple sclerosis patients with different disease phenotypes. PloS One 2017; 12: e0169460.

30. Lunde HMB, Telstad W, Grytten N, et al. Employment among patients with multiple sclerosis - a population study. PloS One 2014; 9: e103317.

31. Findling O, Baltisberger M, Jung SM, et al. Variables related to working capability among Swiss patients with multiple sclerosis - a cohort study. PloS One 2015; 10: e012185.

32. Campbell J, Rashid W, Cercignani M, et al. Cognitive impairment among patients with multiple sclerosis: associations with employment and quality of life. Postgrad Med J 2017; 93: 143-147.

33. Frisell T, Forsberg L, Nordin N, et al. Comparative analysis of first-year fingolimod and natalizumab drug discontinuation among Swedish patients with multiple sclerosis. Mult Scler 2016; 22: 85-93.

34. Lezak MD, Howieson DB, Bigler ED, et al. Neuropsychological Assessment. Oxford: Oxford University Press, 2012.

35. Landfeldt E, Castelo-Branco A, Svedbom A, et al. Personal income before and after diagnosis of multiple sclerosis. Value Health 2018; 21: 590-595.

36. Gyllensten H, Wiberg M, Alexanderson K, et al. How does work disability of patients with MS develop before and after diagnosis? A nationwide cohort study with a reference group. BMJ Open 2016; 6: e012731. 